\title{
Lemoine, A. et P. Sartiaux (2014). Jouer avec les mathématiques: Jeux et activités traditionnels de 2,5 à 8 ans. De Boeck Education, \\ Louvain-la-Neuve \\ doi:10.18162/fp.2016.a109
}

\section{RECENSION}

Dans le but de favoriser l'usage des jeux de mathématiques dès le début du curriculum scolaire, le présent ouvrage vise un rapprochement entre les activités de jeux des jeunes élèves et les savoirs théoriques traitant du développement de la pensée mathématique.Pour ce faire,Lemoine et Sartiaux (2014) proposent aux pédagogues de s'inspirer d'une banque non exhaustive de jeux mathématiques, mise en valeur par une réflexion didactique, afin de piloter leurs activités d'enseignement.

\section{Présentation}

Ce volume est divisé en deux sections, soit : la première concerne les différents jeux mathématiques pouvant être utilisés en milieu scolaire et la viset à décrire quelques aspects théoriques relevant des notions mathématiques mises en lumière dans la première partie du livre.

La première section de l'ouvrage est composée de six chapitres. Le premier met en valeur l'apport de la mise en place des jeux de règles dans le milieu scolaire. Différents points de vue sont exposés afin de justifier l'implantation des jeux dans les salles de classe, soit: des intérêts pédagogiques relevant des dimensions affective, motrice, cognitive, psychologique et socioculturelle. Le second chapitre présente quatre grands types de jeux de règles: le jeu de l'oie, les dominos, le jeu de loto et les casse-têtes. Une suggestion d'adaptation de ces jeux est suggérée aux pédagogues afin de répondre à leurs besoins et intérêts spécifiques. 
Les chapitres trois, quatre et cinq se caractérisent par la présentation de différents matériels de manipulation présents dans les salles de classe ainsi que d'un potentiel usage de ceux-ci permettant d'amorcer des activités propres aux jeux. Le chapitre trois propose des activités en lien avec l'usage d'un matériel logique ou structuré : blocs logiques et vignettes logiques. Le chapitre quatre propose la mise en place de jeux mathématiques à partir de réglettes Cuisenaire, tandis que le chapitre cinq suggère d'utiliser des histoires mathématisées en tant qu'amorces à des jeux traitant de l'arithmétique ou de la géométrie.

Le chapitre six met de l'avant différentes activités à connotation mathématiques pouvant être utilisées à titre d'assises à la mise en place de jeux dans les salles de classe. Des suggestions d'activités psychomotrices, culinaires, manuelles et même des chants sont présentés au sein de ce chapitre.

Ensuite, la seconde section du livre, traitant de la dimension théorique concernant l'usage des jeux de mathématiques, est divisée en cinq chapitres. Les deux premiers chapitres de cette section sont intimement liés. Ceux-ci s'adressent principalement aux pédagogues oeuvrant au préscolaire. Le premier chapitre présente des repères théoriques concernant la construction d'ensembles à partir de différentes caractéristiques associées à un objet. Le chapitre deux est complémentaire au premier. Celui-ci se caractérise par une mise au point théorique à propos des relations envisageables entre les différents éléments d'un ensemble.

Les chapitres trois et quatre approfondissent les fondements didactiques liés à l'apprentissage des nombres et de la géométrie. Enfin, le dernier chapitre de l'ouvrage ne vise pas de domaine particulier des mathématiques. Celui-ci propose quelques repères concernant la progression des activités en mathématiques. Ces propositions visent à distinguer les types d'activités à mettre en place dans le milieu scolaire ainsi qu'à cibler des notions mathématiques à aborder selon l'avancement de l'année scolaire.

\section{Forces et faiblesses de l'ouvrage}

L'ouvrage de Lemoine et Sartiaux (2014) propose différents jeux et activités de mathématiques à mettre en place dans le milieu scolaire. L'œuvre, qui est principalement destinée aux enseignants du préscolaire et du premier cycle du primaire, se caractérise par des chapitres bien écrits et structurés. Par ailleurs, il importe de mentionner que la lecture de la première section peut être pénible à un lecteur peu habitué aux ouvrages relevant de l'enseignement des mathématiques. Cela est essentiellement attribuable au fait que certains éléments théoriques, mentionnés lors de la présentation des jeux, sont seulement mis de l'avant au sein de la seconde section du volume. Sans une appropriation préalable de ces notions, une compréhension approfondie de l'intérêt pédagogique de mettre en place certains jeux en salles de classe peut être ardue. Conscients des difficultés occasionnées, Lemoine et Sartiaux (2014) sont fidèles à la visée principale de l'ouvrage, soit d'offrir aux pédagogues une banque non exhaustive de jeux de mathématiques pouvant être utilisée adaptée en milieu scolaire. Suite à la présentation de la banque de jeux, les liens entre la théorie et la pratique sont effectués, mais ceux-ci appellent la lecture de la seconde section du livre. 
À cet effet, le volet théorique de l'ouvrage est fort bien articulé. Celui-ci risque d'intéresser autant les chercheurs universitaires que les pédagogues oeuvrant au préscolaire ou au primaire. Outre, les concepts tirés de la genèse des structures logiques élémentaires de Piaget et Inhelder (1959), les notions mathématiques ainsi que leur hiérarchisation, présentées sous forme de progression, sont très bien vulgarisés.

Enfin, une force importante de l'ouvrage découle des adaptations diverses proposées pour chacun des jeux. Appuyées sur des fondements théoriques, les modalités d'adaptation des jeux sont, la majorité du temps, décrites soigneusement point par point. Lorsque ce n'est pas le cas, celles-ci sont implicitement mises de l'avant. À mon avis, ces descriptions d'adaptations possibles des jeux octroient aux pédagogues, qui décident de s'approprier l'ouvrage, un sentiment accru de compétence, et ce, en mettant à leur disposition un outil de format "clé en main» pouvant être utilisé selon leurs besoins et intérêts. Conséquemment, pour tout pédagogue qui apprécie les défis de créativité découlant de l'élaboration de jeux ou d'activités de mathématiques, je recommande fermement la lecture du livre de Lemoine et Sartiaux (2014).

\section{Références}

Piaget, J. et Inhelder, B. (1959). La genèse des structures logiques élémentaires ; classification et sériations. Delachaux et Niestlé, Neuchâtel.

\section{Pour citer cet article}

Rajotte, T. (2016). Lemoine, A. et P. Sartiaux (2014). Jouer avec les mathématiques : Jeux et activités traditionnels de 2,5 à 8 ans. De Boeck Education, Louvain-la-Neuve. Formation et profession. 24(3), 104-106. http://dx.doi.org/10.18162/fp.2016.a109 